\title{
Towards Synthesis of Biology and Semiotics
}

\author{
Alexei Sharov ${ }^{1}$ - Timo Maran ${ }^{2} \cdot$ Morten Tonnessen $^{3}$
}

Published online: 4 April 2015

(C) Springer Science+Business Media Dordrecht 2015

The journal Biosemiotics was envisioned by its founding editor, Marcello Barbieri, as a major periodical for interdisciplinary papers that integrate biology and semiotics. Since 2008 the journal has published 21 issues, including special issues on crucial problems such as the semiotics of perception, origins of mind, code biology, biohermeneutics, biosemiotic analysis of information and chance. The impact factor of the journal (currently 0.488) does not fully describe the significance of this journal, because the discipline of biosemiotics is young and remains in its early phase of growth. As the new editorial team of Biosemiotics, we would like to express our gratitude to Dr. Barbieri for his excellent job as an editor, and ensure the readers that we are equally committed to maintain high standards and the scientific rigor of published papers. At the end of 2014 we reorganized the editorial board of the journal based on the credential and former activity of prospective members. The current list of 28 members can be found on the cover page. We want to thank members of the former editorial board for their support and work. In the following we introduce our vision of the state and future of biosemiotics and its representation in this journal.

Biosemiotics is dedicated to building a bridge between biology, philosophy, linguistics and the communication sciences. According to the Oxford Dictionary of Biochemistry and Molecular Biology (Smith et al. 1997: 72) biosemiotics is "the study of signs, of communication and of information in living organisms". However, we think that biosemiotics has acquired a more general scope. Today, its main challenge is the attempt to naturalize not only biological information (genetic, epigenetic, sensorial, and behavioral) but also biological meaning. The main idea of biosemiotics is that life and semiosis are coextensive. Here semiosis is understood as a sign process or sign exchange, where signs stand for something else in some respect or capacity. In particular, life has a semiotic nature because it is based on endless interpretation of

Alexei Sharov

sharoval@mail.nih.gov

1 National Institute on Aging, Baltimore, MD, USA

2 Department of Semiotics, University of Tartu, Tartu, Estonia

3 University of Stavanger, Stavanger, Norway 
environmental cues and transfer of life-related functional meanings vertically across generations and horizontally to neighboring organisms. Semiotic processes help organisms to perform their functions, preserve their habits and pursue their agendas through generations (Hoffmeyer 1996, 2008; Sharov 1992). One implication of this view is that life has certain mind-like properties that enable meaningful interpretation of perceived signs; this outlook contrasts with the traditional dualistic approach in science that distinguishes sharply between mind and matter. Biosemiotics, in comparison, is an evolutionary science because it explores the gradual emergence of each single quality, including broad phenomena such as life and mind. The mind-like properties found elsewhere in nature can be very different from the human mind. Biosemiotics does not promote anthropomorphism, but acknowledges the diversity of semiosis across and within the various levels of biological organization. It also differs from radical monistic theories (e.g., physiosemiotics or pansemiotics), which overemphasize the transitional nature of changes to the extent that qualitative differences in effect disappear.

Although some scholars consider biosemiotics predominantly as a philosophy, particularly within philosophy of biology/science, we would like to emphasize its scientific and often practical orientation. Biosemiotics can contribute to natural science, applied science and philosophy alike. Our scientific outlook does not mean, however, that we support mechanistic methodology, which currently dominates in physics, chemistry, and even in molecular biology. We believe that traditional approaches in science associated with studies of passive, isolated systems, as in physics and chemistry, should be complemented by a distinctively different study of complex agents such as living organisms and their components, people, human organizations, and technological artifacts. The radically different nature of these complex phenomena requires a substantial shift in scientific methodology. Biosemiotics considers the existence of entities that are not directly accessible for investigation (e.g., meaning, agency, goals, internal representations). It therefore seeks to identify or develop indirect methods that can help to evaluate these entities, and supports systematic approaches for their analysis. These efforts should be based on diverse heuristics, handling of multiple hypotheses, and complementarity of different descriptions as exemplified by the brief overview of papers presented in this issue (see below).

We believe that biosemiotics will reshape both biology and semiotics. Our field is not simply an application of existing semiotic theories in the area of biology, nor is it a "renaming game" within biology. Biology needs to recognize the semiotic nature of life and reformulate its theories and methodologies accordingly. In particular, many core concepts in biology (e.g. code, translation, signal transduction) are inherently semiotic. Recent developments in evolutionary developmental biology, molecular biology, and epigenetic studies have made the gene-centric view of evolution (also known as genetic determinism) largely obsolete, and biologists should aim for a new consensus on such terms as "coding", "heritability", and "acquired character". For example, learned behaviors may be heritable even if they are not encoded in the genome (Sharov 2014). Biology should also recognize the enormous hidden functional capacities of organisms, which fascinated many biologists in the past. For example, Hans Driesch discovered that individual blastomere cell isolated from an early embryo can develop into a full animal. Goldschmidt described systemic and highly non-random heritable variations where organs developed in unusual locations of the body. Vavilov described repeated series of variation in several lineages of plants, which indicated 
common internal capacities to generate specific morphologies. Unfortunately, these studies were dismissed by neo-Darwinism for decades because they contradicted the assumed dogma of random and blind variation. Thus, we expect that biological theory will change substantially as a result of its integration with semiotics.

Similarly, substantial changes should be expected in semiotics. Semiotics was initially, and are by many still conceived of as a branch of linguistics, which is applied exclusively to human communication. The principles of semiotics were later used to describe the behavior of animals with brains, but only a few semioticians accepted the idea that signification (i.e., the capacity of agents to use signs as guides for activity) is the fundamental property of all living organisms. One of the major propagators of the biosemiotic movement was Thomas A. Sebeok (2001). The semiotic enterprise's expansion to the entire tree of life is an enormous challenge. It is naïve to expect that human-centered or brain-centered semiotic theories will work at all levels of biological hierarchy. We have to admit that semiosis in bacteria is qualitatively different from the semiosis in eukaryotic cells, and even more radically different from the brain-based semiosis of animals. Semiosis evolved with and in life, and both life and semiosis gradually increased their complexity and organization. This process of evolution is not merely a quantitative affair, but a process paved with important qualitative inventions that at some points in time restructured both the nature of life and the character of signification. Focusing on the diverse forms of semiosis in the natural world implies, among other things, emphasizing the role of prelinguistic sign processes in humans, and scrutinizing their relations with language as well as their relations with nonlinguistic semiosis in other species.

The journal Biosemiotics is the official periodical of the International Society for Biosemiotic Studies (ISBS), and the policy of the journal is discussed in Editorial board meetings during the annual Gatherings in Biosemiotics. We welcome contributions from all relevant disciplines, including biology, philosophy, semiotics, cognitive science, information theory, linguistics, anthropology, ecology, and behavioral sciences. However, biological studies should explicitly discuss semiotic processes and, in one form or another, the meaning of biological information. In the same way, linguistic or cognitive studies, for example, should explicitly and extensively discuss the biological aspects of communication and cognition. In all cases, no matter what the angle, we seek exploration of the synthesis of biology and semiotics and expect analysis of relevant biosemiotic literature. Original research papers should include novel ideas that are explicitly stated in the abstract and subsequently proved, or rigorously argued for, in the main body of text. We expect papers to follow a well-designed, logical structure, where all sections follow each other logically, and each theoretical statement is defended and illustrated with relevant examples. We do not encourage publication of merely philosophical essays, because this style of reasoning, suitable as it may be in other contexts, does not provide sufficient scientific detail and relevance. In order to be relevant for Biosemiotics, in other words, philosophical contributions must merge with biological reasoning.

We would also like to mention some technical issues related to publication in Biosemiotics. Most important is that all operations (e.g., manuscript submission and peer-review) are now carried out in an automated fashion through the Editorial Manager web program. We kindly ask all contributors and reviewers to use this electronic system. A tutorial is available at the homepage of the Editorial Manager 
(www.editorialmanager.com/bise). In case of any problems, do not hesitate to contact us and ask for help. Another issue is that the duties of the Editor-in-Chief are shared by three people, which may be confusing. We would like to disclose that all decisions about papers are discussed by all three editors, unless some editor is excluded due to direct involvement (e.g., as an author or supervisor of an author). In most cases, our decisions are made unanimously.

Here is a short overview of ideas that we have started implementing in order to promote biosemiotic studies and facilitate publications in our journal: First, we organize special issues (approximately one per year) that are focused on some of the most important or provoking, novel ideas. Currently, Jesper Hoffmeyer is editing a special issue on "Semiotic Scaffolding" which will be published as issue \#2 2015. Moreover, Luis E. Bruni has agreed to organize a special issue that is focused on "Multi-Level Semiosis", to be published in 2016. Second, the journal helps the readers to navigate in current literature by publishing subject reviews and book reviews. For example, John Coletta has written a review of the book "The Logos of the Living World: MerleauPonty, Animals, and Language" by Westling, and Lucie Čadková has conducted a review of Hockett's theory of language. We also encourage authors to use the format of "review papers" to write overviews on essential and well-focused topics in biosemiotics. Related to the genre of the review article, Morten Tønnessen has developed a methodology for mapping and evaluating the actual use of biosemiotic terms via questionnaires and analysis of literature. His review article on the meaning of the terms "agent" and "agency" is presented in the current issue (see below). We envision that similar review articles will be published regularly, usually with one such review article per regular issue. En masse they will comprise the "Biosemiotic Glossary Project", which we plan to facilitate over the next few years. Morten Tønnessen is the handling editor of the review articles in the Biosemiotic Glossary Project. The assigned authors are tasked with conducting their reviews of selected terms in an objective manner, but are simultaneously allowed to suggest innovative synthesis in term usage. The Biosemiotic Glossary Project thus aims to document historical and current term usage in biosemiotics, and moreover to contribute to further theoretical development. This series of review articles is a community effort, as reflected in our established procedures which in addition to the open survey involves routines for incorporating feedback from the Editorial board and from those cited in the respective review articles.

Another initiative is the establishment of the Annual Biosemiotic Achievement Award, in cooperation with ISBS and Springer. The purpose of the award, which will go to the best article published in each volume (i.e. each year), is to promote biosemiotic research, its scientific impact and future prospects. ${ }^{1}$ The very first prize will be awarded based on papers in the 2015 volume. Award winners will be presented in \#1 of each volume, as well as on the ISBS webpage and in the ISBS annual general meeting (held at Gatherings in biosemiotics). While the Biosemiotic Glossary Project aims to promote theoretical reflection and development in the biosemiotic community, the Annual Biosemiotic Achievement Award seeks to encourage novelty in terms of theoretical or empirical discovery (i.e., enhanced description and explanation of existing and/or discovery of new

\footnotetext{
${ }^{1}$ The statutes of the award are available at http://biosemiotics.org/statutes_of_the_annual_biosemiotic_ achievement_award.pdf.
} 
empirical phenomena). We hope the award can contribute to making our field more visible, and to attract more submissions to Biosemiotics.

The current issue of Biosemiotics includes several papers that discuss the phenomenon of language, its features, and scope. Traditionally, the term "language" was applied narrowly to the human species, and Hockett pioneered delineating unique features of human language that distinguish it from animal communication. But as demonstrated in recent studies, some forms of animal communication appear to be more complex than previously thought of, and may, depending on the exact notion of language, qualify to be considered as language. Čadková advocates an inclusive approach where most types of communication in animals, even in insects (e.g. the dance of bees), are viewed as languages. Her claim is that the languages of different species differ mostly quantitatively rather than qualitatively. In particular, she supports the suggestion of Slobodchikov and Segerdahl that Hockett's emphasis on the acoustic channel and articulated speech is not relevant as a "design feature" of language. Similarly, Wacewicz and Żywiczyński argue that Hockett's design features cannot support the theoretical framework for evolutionary linguistics, and that the contemporary understanding of human language has developed to be very different from that of Hockett's studies in the 1950ties. In particular, they hold that language should be seen in light of the sensorial, cognitive, and social abilities that enable the use of language. The theory of language, in their mind, should not be limited to means of communication, but should also address the cognitive abilities of language users.

Sukhoverkov and Fowler expand the ideas of the 'Extended evolutionary synthesis' so as to account for the evolution of language. In particular, they consider the role of different types of memory and non-genetic inheritance for the origin and evolution of language. They consider language and memory as distributed, heteronomous systems that integrate the biological and social domains. In their view, the logical structure of languages includes the inductive systems that are responsible for adjusting living processes to the environment, and deductive systems that are inherited and depend on the internal development of individuals and societies. The origin, retention, and evolution of any natural sign system, then, presuppose the existence of supporting memory structures (e.g. physical, genetic, individual/neural, or social). Advanced sign systems are furthermore reinforced and maintained by conventional and deliberate social regulation and accumulation.

Cole develops an entirely different approach to language by focusing on qualitative features that separate human language from animal communication, and finds support for his ideas in archeological records. Following Deacon, Kull and Barbieri, he assumes that animal communication is based solely on icons and indexes, whereas human communication is based on symbols that have abstract meanings established via social conventions. Another qualitative difference, he claims, comes from the theory of mind: the behavior of animals is guided by low-level intentionality (first- and second-order only), but humans use high-level intentionality by anticipating intentional and intelligent actions from other individuals. Finally, Cole develops the notion of agent identity (which includes at least seven categories) as a dialectic way of integrating an individual with its social environment. He writes: "The identity model allows a correlation between a scale of cognitive acuity, hominin behaviour through the archaeological record and subsequently language development within an evolutionary context." In particular, he views standardization of tools as an indicator of social conventions and 
symbolic communication. The use of the theory of mind and components of identity is a welcome addition to our understanding of language that goes beyond the traditional linguistic analysis. However, Cole's claim that animals lack symbols and higher-level intentionality in their behavior and communication might seem controversial. It is well known that even bees can develop abstract concepts of object shape (not dependent on size) and object number (not dependent on shape). Mammals and birds can learn new social behaviors and participate in complex communication with other animals and with humans. Anticipation of intentionality in others is seen for instance in bait-fishing behavior known in herons, gulls, crows and other birds, where a bird drops an object to the water in order to lure and catch fish (Ruxton and Hansell 2011).

The papers by Fernandez and Sharov and Vehkavaara represent different paths towards integration of semiotics and biology. Both views are antithetical to the neoDarwinian stance because they assume the emergence of novelty as a source of evolutionary change. According to neo-Darwinism, natural selection is the only factor of innovation, whereas organisms are mere passive tokens that are mechanically copied with errors. Fernandez follows Peirce's view that logic is embedded in the world, and proposes that biological evolution can be explained as "concrete generalization", which is similar to the conceptual generalization in logic. Just as logic helps to construct new concepts from old ones, biological evolution constructs new forms and types as modifications and combinations of old forms and types. Thus, Fernandez concludes that "[s]emiosis and biological evolution are two sides of the same coin." Concrete generalization is driven by semiotic causation, which is brought about by the action of signs upon appropriate receptive structures, and is largely independent from the energy of the signal. Semiotic modulation (or regulation) produces a dynamic replica of a given general form (or type). Thus, biological reproduction is seen as analogous to sign copying, although the former process is much more complex than the latter one. These ideas are then illustrated through comparisons between the evolution of organisms and that of human artifacts.

In contrast to the "logic-first" Peircean approach, Sharov and Vehkavaara focus on the functional aspect of biological evolution, where sign relations and logic are tools constructed by evolving living agents in order to perform living functions, survive, and reproduce. They reject Peirce's idea that signs exist on their own, independently of agents/interpreters, and propose (following Uexküll) to view signs as they are perceived and interpreted by each kind of agents. Each species of animals, they stress, has its own model of the world (Umwelt) that is used to interpret external signals and support communication between individuals. The capacity to perceive and classify objects, in particular, is limited in primitive organisms (e.g., in ticks), they claim. Sharov and Vehkavaara argue that most simple organisms, such as bacteria, entirely lack this capacity and associate signs directly with actions rather than with objects. They call this kind of semiosis "protosemiosis", following Prodi, who applied this notion to molecular processes in the cell. In advanced forms of semiosis, or "eusemiosis", on the other hand, agents associate signs with objects and only then possibly with actions. Eusemiosis is qualitatively different from protosemiosis because it cannot be reduced to a small number of specific signaling pathways. Proto-signs can be classified as protoicons that signal via single specific interaction, proto-indexes that combine several functions, and proto-symbols that are processed by a universal subagent equipped with a set of heritable adapters. In summary, protosemiosis is a primitive kind of semiosis 
that supports "know-how" without "know-what" - and without studying protosemiosis, biosemiotic theory would be incomplete.

In the first review article in the Biosemiotic Glossary Project, Tønnessen reviews the terms 'agent' and 'agency' as these are used in the biosemiotic community. The article is intended as a standard-setting article that establishes the format for future articles in the Biosemiotic Glossary Project. By describing differences in biosemiotic term usage in a neutral manner, it demonstrates the value of such broad, open surveys as the article is itself based on. While there is no consensus in the biosemiotic community on the terms 'agent' and 'agency', Tønnessen concludes that most biosemioticians appear to agree that core attributes of an agent include goal-directedness, self-governed activity, processing of semiosis and choice of action, with these features being vital for the functioning of the living system in question. He observes that it is legitimate to refer to a specifically biosemiotic notion of agency, but underlines that such a notion does not cover all usages of the terms 'agent' and 'agency' among biosemioticians.

Taken as a whole, the articles of this issue map the complexity and diversity of sign processes from the threshold of language to protosemiosis, with implications and perspectives for further theoretical development of biosemiotics.

\section{References}

Hoffmeyer, J. (1996). Signs of meaning in the universe. Bloomington: Indiana University Press.

Hoffmeyer, J. (2008). Biosemiotics: An examination into the signs of life and the life of signs. Scranton: University of Scranton Press.

Ruxton, G. D., \& Hansell, M. H. (2011). Fishing with a bait or lure: a brief review of the cognitive issues. Ecology, 117(1), 1-9.

Sebeok, T. A. (2001). Signs, bridges, origins. In T. A. Sebeok (Ed.), Global semiotics (pp. 59-73). Bloomington: Indiana University Press.

Sharov, A. A. (1992). Biosemiotics: functional-evolutionary approach to the problem of the sense of information. In T. A. Sebeok \& J. Umiker-Sebeok (Eds.), Biosemiotics. The semiotic web 1991 (pp. 345-373). New York: Mouton de Gruyter.

Sharov, A. A. (2014). Evolutionary constraints or opportunities? Biosystems, 120, 21-30.

Smith, A. D., Data, S. P., Smith, G. H., Campbell, P. N., Bentley, R., \& McKenzie, H. A. (Eds.). (1997). Oxford dictionary of biochemistry and molecular biology. Oxford: Oxford University Press. 